\title{
Long-term follow-up of complete remission of advanced hepatocellular carcinoma following sorafenib therapy: A case report
}

\author{
JUNG GIL PARK ${ }^{1}$, WON YOUNG TAK ${ }^{2}$, SOO YOUNG PARK ${ }^{2}$, YOUNG OH KWEON ${ }^{2}$, SE YOUNG JANG ${ }^{2}$, \\ SOO HYUN LEE ${ }^{2}$, YU RIM LEE ${ }^{2}$, SUN KYUNG JANG ${ }^{2}$, KEUN HUR ${ }^{3}$ and HEON JU LEE ${ }^{1}$ \\ ${ }^{1}$ Division of Gastroenterology and Hepatology, Department of Internal Medicine, College of Medicine, Yeungnam \\ University; ${ }^{2}$ Division of Gastroenterology and Hepatology, Department of Internal Medicine; ${ }^{3}$ Department of Biochemistry \\ and Cell Biology, School of Medicine, Kyungpook National University, Daegu 41940, Republic of Korea
}

Received March 30, 2016; Accepted June 15, 2017

DOI: $10.3892 / 01.2017 .6788$

\begin{abstract}
Sorafenib is a tyrosine kinase inhibitor that has been demonstrated to improve the overall survival time of patients with advanced hepatocellular carcinoma (HCC). Although there have been a number of reports of patients achieving complete remission (CR) following sorafenib therapy, the long-term clinical outcomes of these patients have yet to be ascertained. A 72-year-old male patient with chronic hepatitis $\mathrm{C}$, diabetes, hypertension and an old cerebral infarction was referred for the evaluation of a liver mass identified on an abdominal ultrasound. Abdominal computed tomography (CT) demonstrated a $13-\mathrm{cm}$ mass replacing the right lobe of the liver, with portal vein thrombosis. HCC was confirmed by a percutaneous needle biopsy and treated with sorafenib. At 4 months, a follow-up CT demonstrated no enhancing viable lesions in the tumor and recanalization of the portal vein. Sorafenib therapy was continued for 48 months until the patient experienced dyspnea due to congestive heart failure, with pleural effusion. Following the discontinuation of sorafenib, the patient's symptoms improved. The patient followed up without recurrence for 52 months. Subsequent to achieving CR through treatment with sorafenib, long-term sorafenib therapy may be an option and efforts should be made to monitor cardiac toxicity during sorafenib therapy, particularly in high-risk patients.
\end{abstract}

Correspondence to: Dr Won Young Tak, Division of Gastroenterology and Hepatology, Department of Internal Medicine, School of Medicine, Kyungpook National University, 130 Dongdeok-ro, Jung-gu, Daegu 41940, Republic of Korea

E-mail:wytak@knu.ac.kr

Abbreviations: HCC, hepatocellular carcinoma; VEGFR, vascular-endothelial grow th factor receptor; SHARP, sorafenib HCC assessment randomized protocol trial; $\mathrm{CR}$, complete remission; ALT, alanine aminotransferase; AFP, $\alpha$-fetoprotein

Keywords: sorafenib, complete remission, hepatocellular carcinoma, chemotherapy, cardiotoxicity

\section{Introduction}

Hepatocellular carcinoma (HCC) is the third-leading cause of cancer-associated mortality worldwide, accounting for more than 600,000 deaths per year (1). A large proportion of these patients are not candidates for potentially curative therapy, including surgical resection, liver transplantation or local ablation therapy, as they are diagnosed with advanced disease with vascular invasion and/or distant metastasis $(2,3)$. Sorafenib is a multi-kinase inhibitor, which inhibits a number of kinase-dependent signaling pathways associated with tumor progression and angiogenesis, including those involving Raf serine/threonine kinase, vascular endothelial growth factor receptor (VEGFR)-2, VEGFR-3, and platelet-derived growth factor $\beta$-receptor $(4,5)$. The efficacy of sorafenib was demonstrated in two large phase III clinical trials including the Sorafenib HCC Assessment Randomized Protocol (SHARP) trial and the Asia-Pacific trial (conducted in the Asia-Pacific region) $(6,7)$. Although there was a survival benefit for patients with advanced HCC, only a limited number of patients demonstrated a partial response, and there were no cases of complete remission (CR). Furthermore, the marginal observed survival gain of 2.8 months in the SHARP trial and 2.3 months in the Asia-Pacific trial did not meet the expectations of clinical practice. Following the approval of sorafenib, several cases of CR subsequent to sorafenib therapy were reported worldwide (8-11). However, there is little data regarding the clinical course and safety of long-term sorafenib therapy in cases of $\mathrm{CR}$. Therefore, the present study reports a case of long-term maintenance therapy with sorafenib following the achievement of CR in a patient with advanced HCC.

\section{Case report}

A 72-year-old male patient was referred for the evaluation of a liver mass identified on an abdominal ultrasound performed for routine health screening purposes. The patient was taking medications for diabetes, hypertension and an old cerebral infarction. Initial laboratory results were as follows: White blood cells $5,920 / \mathrm{mm}^{3}$ (normal range, $4-10 \times 10^{3} / \mathrm{mm}^{3}$ ), 
hemoglobin level $14.0 \mathrm{~g} / \mathrm{dl}$ (normal range, 12-16 g/dl), platelet count $237 \times 10^{3} / \mathrm{mm}^{3}$ (normal range, $100-300 \times 10^{3} / \mathrm{mm}^{3}$ ), alanine aminotransferase (ALT) 29 IU/1 (normal range, 0-40 IU/1), total bilirubin $1.0 \mathrm{mg} / \mathrm{dl}$ (normal range, $0.1-1.1 \mathrm{mg} / \mathrm{dl}$ ), albumin $3.9 \mathrm{mg} / \mathrm{dl}$ (normal range, $4.0-5.5 \mathrm{mg} / \mathrm{dl}$ ) and prothrombin time $11.6 \mathrm{sec}$ (normal range, 10.4-13.3 sec). With regard to virological markers, the patient was positive for hepatitis $C$ virus antibody and negative for hepatitis B surface antigen. The serum $\alpha$-fetoprotein (AFP) level and protein induced by vitamin $\mathrm{K}$ absence or antagonist-II level were $60,500 \mathrm{ng} / \mathrm{ml}$ (normal range, $0-1 \mathrm{ng} / \mathrm{ml}$ ) and 2,000 $\mathrm{mAU} / \mathrm{ml}$ (normal range, 0-40 mAU/ml), respectively. Baseline electrocardiography (ECG) and echocardiography revealed normal left ventricular systolic function (left ventricular ejection fraction, 65\%) with normal sinus rhythm. Multiphasic computed tomography (CT) scans of the abdomen demonstrated a huge, $13 \mathrm{~cm}$-sized mass replacing the right lobe of the liver, with portal vein thrombosis (Fig. 1A). HCC was confirmed by ultrasound-guided percutaneous needle biopsy of the liver mass (Fig. 2). The cancer was staged as advanced HCC according to the Barcelona Clinic Liver Cancer staging system (12) and the patient's Eastern Cooperative Oncology Group performance status (13) was 1; the patient was treated with $400 \mathrm{mg}$ sorafenib (Nexavar; Bayer AG, Leverkusen, Germany) twice daily. Following the initiation of sorafenib therapy, the patient experienced a grade 1 (14) hand-foot skin reaction, which was well tolerated without requiring a dose reduction.

After 40 days of sorafenib therapy, the tumor size had decreased to $6 \mathrm{~cm}$ and the serum AFP level had decreased to $571 \mathrm{ng} / \mathrm{ml}$. The follow-up abdominal CT scans at 4 months demonstrated no enhancing viable lesions in the tumor and recanalization of the portal vein; additionally, serum AFP values normalized $(8.42 \mathrm{ng} / \mathrm{ml}$; Fig. 1B). The sorafenib dose was maintained at $400 \mathrm{mg}$ twice daily for 16 months, after which the sorafenib dose was reduced to $400 \mathrm{mg}$ a day due to the elevation of serum ALT (231 IU/ml). Sorafenib therapy was continued for 48 months until the patient experienced dyspnea due to congestive heart failure with pleural effusion (Fig. 3). An echocardiogram revealed decreased left ventricular systolic function (left ventricular ejection fraction, 40\%), including new-onset atrial fibrillation on the follow-up ECG. Following the discontinuation of sorafenib and the addition of rate control medication, the patient's dyspnea improved, with the disappearance of the pleural effusion. Without sorafenib therapy, the patient remained in a state of CR, followed up with serial CT scans and tumor marker measurement, for 52 months from the time of initiation of the sorafenib therapy (Fig. 1C). Written informed consent was obtained from the patient for the publication of their clinical data and images.

\section{Discussion}

At present, sorafenib is the only chemotherapeutic agent that has been proven to increase overall survival time in patients with advanced HCC $(6,7)$. Although partial response was achieved in only 2 and $3.3 \%$ of patients in two large phase III clinical trials, with no cases of CR $(6,7)$, there have been several reports of $\mathrm{CR}$ following sorafenib therapy in patients with advanced HCC since sorafenib became available for general clinical practice (8-11). The majority of CR cases

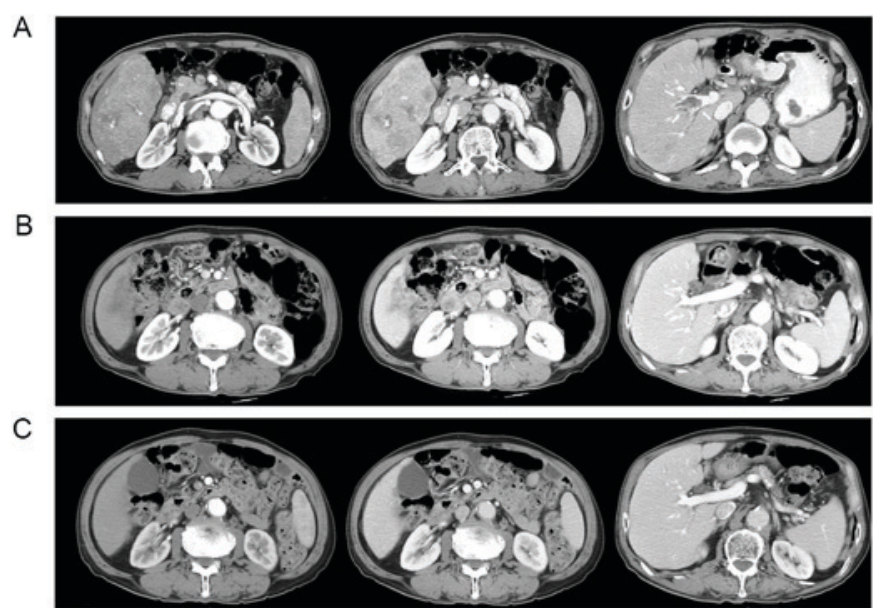

Figure 1. Multiphasic contrast-enhanced CT of the abdomen. (A) Initial abdominal CT scan demonstrated a $13-\mathrm{cm}$ mass with arterial enhancement and early wash-out accompanying tumor thrombus of the portal vein in segment 5 and 6; (B) Following 4 months of sorafenib therapy, CT scans revealed the disappearance of the enhanced mass and recanalization of the portal vein; (C) At the 52 months-follow-up, CT scans demonstrated the further decrease of a non-enhanced mass with a patent portal vein. CT, computed tomography.

exhibited early tumor response following sorafenib therapy accompanied by a rapid radiological response and the normalization of AFP within 6 months of sorafenib therapy (8-11). In accord with previous reports, the present case exhibited a similar clinical course, including a rapid radiologic response and the normalization of serum AFP within 40 days of sorafenib therapy (Fig. 4). Early AFP response following the initiation of sorafenib therapy has previously been identified as a positive prognostic factor for patients with advanced HCC (15). Further investigation regarding the contributing factors and molecular pathophysiology of these sorafenib responders is required.

Although CR cases following sorafenib therapy have been reported in the literature, there is limited data regarding the long-term clinical course of these cases following the achievement of CR (8-11). Therefore, there is no consensus on a management strategy for CR-achieving patients. As in the present case, if the patient can tolerate sorafenib therapy, long-term sorafenib therapy may be an option as a maintenance treatment following CR. However, in addition to the economic burden of continuing sorafenib, there is limited data on safety profiles in regard to long-term sorafenib therapy $(16,17)$. As it targets multiple angiogenic receptors, sorafenib causes a unique spectrum of adverse events including skin rash, stomatitis and cardiovascular toxicity. During sorafenib therapy, cardiovascular adverse events, including hypertension, bleeding and cardiac or cerebrovascular events were among the most serious observed in clinical trials (6). Among these cardiovascular toxicities, hypertension and bleeding are most frequent adverse events associated with sorafenib therapy (18). Although there have been reports of cardiotoxicity from the long-term sorafenib therapy of patients with renal cell cancer and lung cancer, the present case is the first report of cardiotoxicity caused by long-term sorafenib therapy in a patient with advanced HCC; this adverse effect has been underestimated by phase III studies due to the relatively 
A

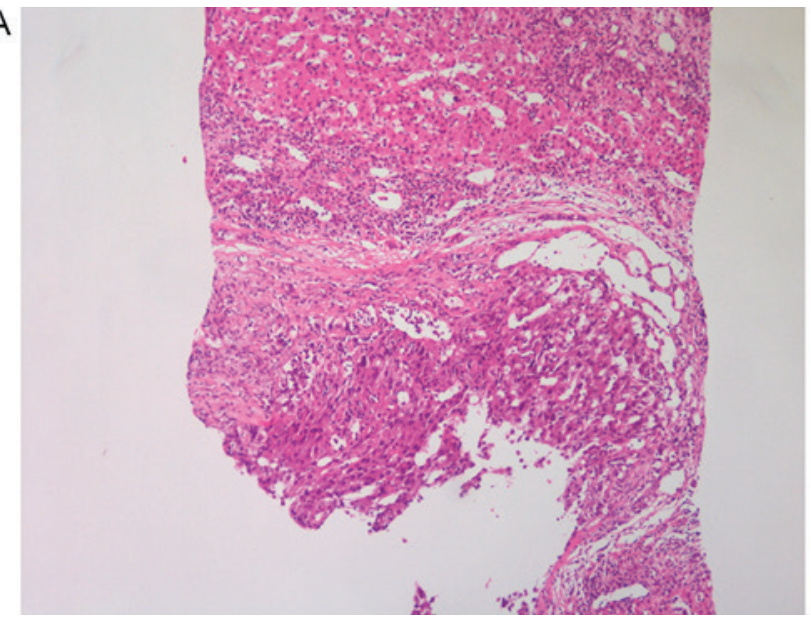

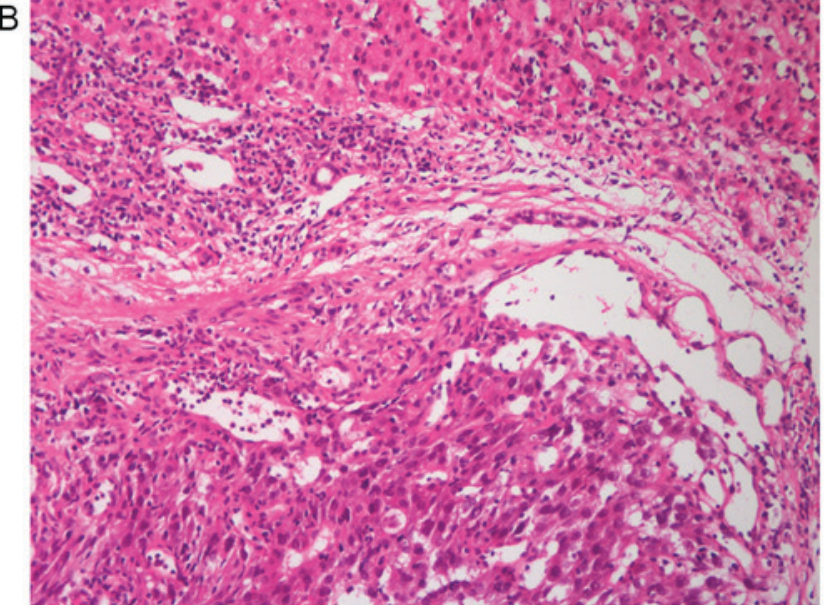

Figure 2. Percutaneous liver biopsy confirmed an Edmondson-Steiner grade III hepatocellular carcinoma featuring a trabecular pattern. (A) HE staining; magnification, x100; (B) HE staining; magnification, x200. HE, hematoxylin and eosin.

A

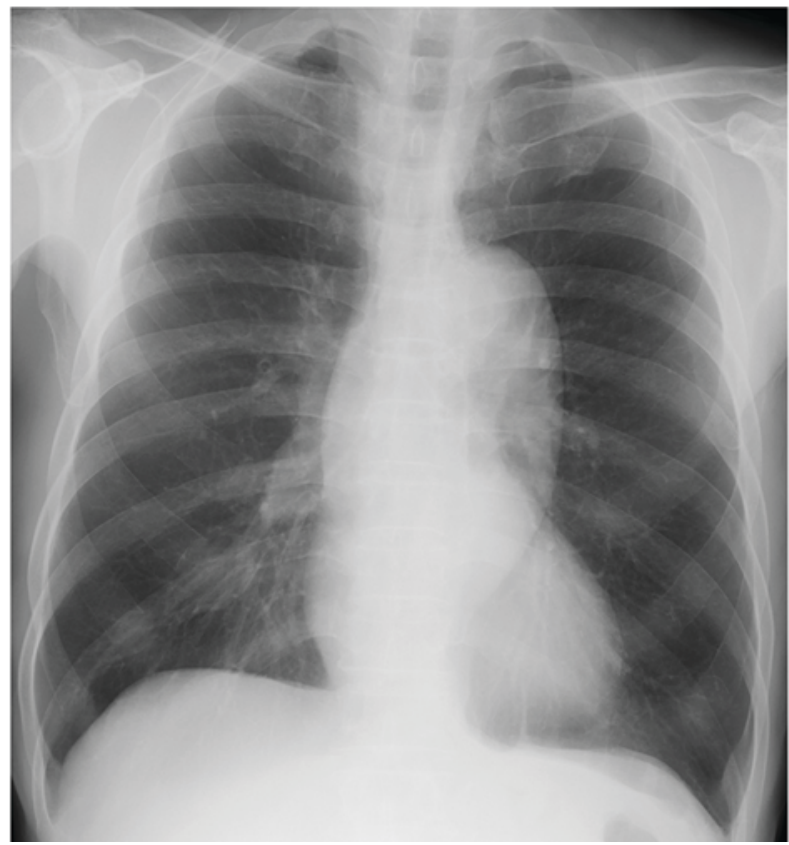

B

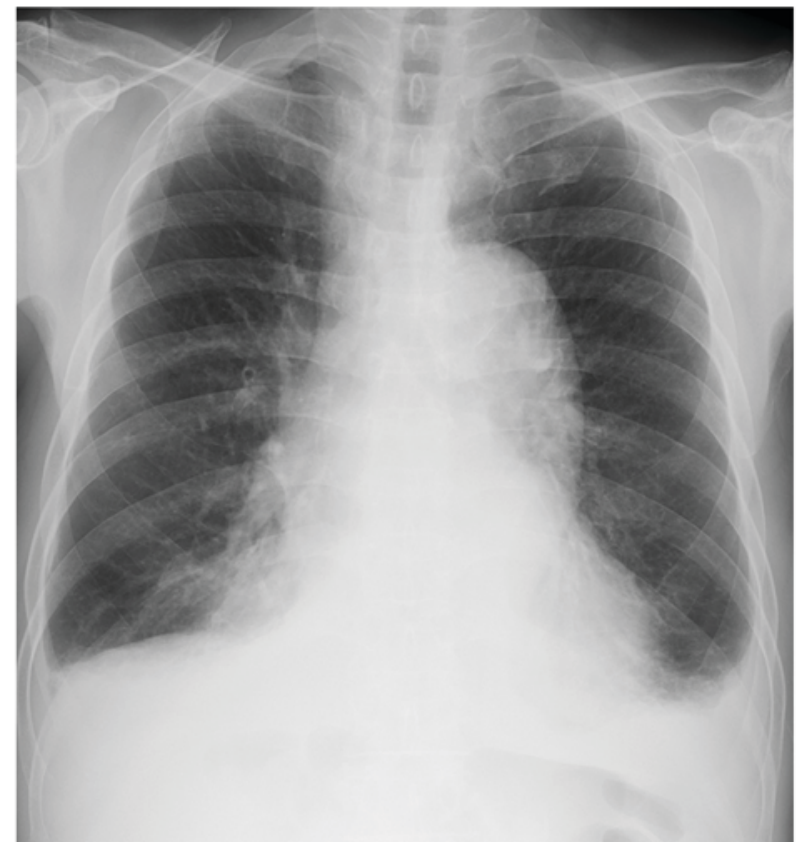

Figure 3. Following 44 months of sorafenib therapy, a follow-up chest x-ray revealed an increase of the pulmonary vascular marking and cardio-thoracic ratio, with left pleural effusion. (A) Initial chest x-ray; (B) Chest x-ray from the 44 month follow-up.

short-term treatment $(19,20)$. The present case encourages the clinician to monitor cardiac toxicity during sorafenib therapy, particularly in elderly patients with underlying cardiovascular diseases.

Compared with the literature to date, the present case represents an HCC case with the longest follow-up subsequent to CR induced by sorafenib therapy. Although the therapeutic response was assessed by radiologic findings, rather than by pathologic confirmation, CR was confirmed by long-term sustained radiologic response with a normal AFP level, even subsequent to the termination of sorafenib therapy. The present case provides the valuable insight that long-term sorafenib therapy may be an option subsequent to achieving CR through treatment with sorafenib, and that efforts should be made to monitor for the appearance of less common adverse events in patients receiving long-term sorafenib therapy.

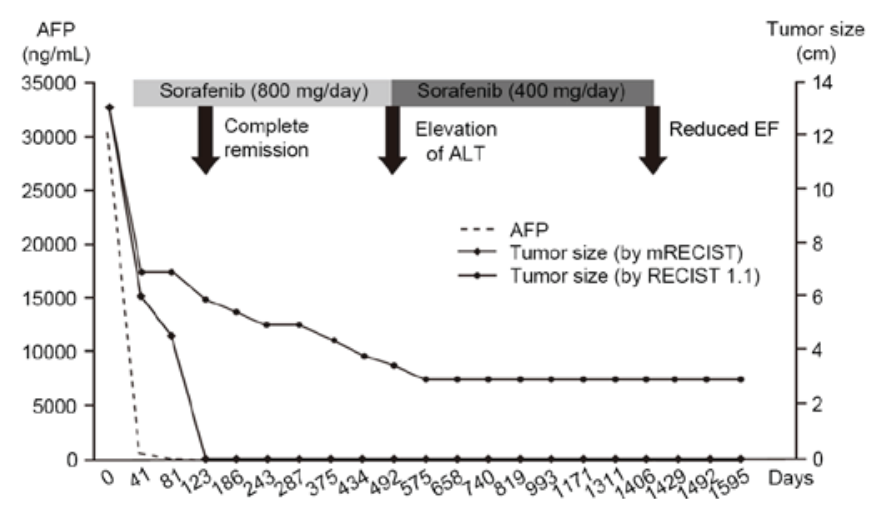

Figure 4. Clinical course and alteration of serum AFP level and tumor size based on RECIST and mRECIST. AFP, $\alpha$-fetoprotein; RECIST, response evaluation criteria in solid tumor; mRECIST, modified response evaluation criteria in solid tumor; ALT, alanine aminotransferase; EF, ejection fraction. 


\section{Acknowledgements}

The present study was supported by the Kyungpook National University Research Fund, 2010 (grant no., 201014100000).

\section{References}

1. Parkin DM, Bray F, Ferlay J and Pisani P: Global cancer statistics, 2002. CA Cancer J Clin 55: 74-108, 2005.

2. Llovet JM, Burroughs A and Bruix J: Hepatocellular carcinoma. Lancet 362: 1907-1917, 2003.

3. European Association For The Study Of The Liver1; European Organisation For Research And Treatment Of Cancer: EASL-EORTC clinical practice guidelines: Management of hepatocellular carcinoma. J Hepatol 56: 908-943, 2012.

4. Wilhelm SM, Carter C, Tang L, Wilkie D, McNabola A, Rong H, Chen C, Zhang X, Vincent P, McHugh M, et al: BAY 43-9006 exhibits broad spectrum oral antitumor activity and targets the RAF/MEK/ERK pathway and receptor tyrosine kinases involved in tumor progression and angiogenesis. Cancer Res 64: 7099-7109, 2004.

5. Wilhelm SM, Adnane L, Newell P, Villanueva A, Llovet JM and Lynch M: Preclinical overview of sorafenib, a multikinase inhibitor that targets both Raf and VEGF and PDGF receptor tyrosine kinase signaling. Mol Cancer Ther 7: 3129-3140, 2008.

6. Llovet JM, Ricci S, Mazzaferro V, Hilgard P, Gane E, Blanc JF, de Oliveira AC, Santoro A, Raoul JL, Forner A, et al: Sorafenib in advanced hepatocellular carcinoma. N Engl J Med 359: 378-390, 2008

7. Cheng AL, Kang YK, Chen Z, Tsao CJ, Qin S, Kim JS, Luo R, Feng J, Ye S, Yang TS, et al: Efficacy and safety of sorafenib in patients in the Asia-Pacific region with advanced hepatocellular carcinoma: A phase III randomised, double-blind, placebo-controlled trial. Lancet Oncol 10: 25-34, 2009.

8. Ahn SY, Lee HS, Kweon YO, Tak WY and Park SY: Sustained remission over 36 months of advanced hepatocellular carcinoma after short-term sorafenib therapy. Dig Dis Sci 58: 1428-1432, 2013.

9. Park JG: Long-term outcomes of patients with advanced hepatocellular carcinoma who achieved complete remission after sorafenib therapy. Clin Mol Hepatol 21: 287-294, 2015.
10. Kim MS, Jin YJ, Lee JW, Lee JI, Kim YS, Lee SY and Chae MH: Complete remission of advanced hepatocellular carcinoma by sorafenib: A case report. World J Gastrointest Oncol 5: 38-42, 2013.

11. Liu D, Liu A, Peng J, Hu Y and Feng X: Case analysis of complete remission of advanced hepatocellular carcinoma achieved with sorafenib. Eur J Med Res 20: 12, 2015.

12. Bruix J and Llovet JM: Prognostic prediction and treatment strategy in hepatocellular carcinoma. Hepatology 35: 519-254, 2002.

13. Oken MM, Creech RH, Tormey DC, Horton J, Davis TE, McFadden ET and Carbone PP: Toxicity and response criteria of the Eastern Cooperative Oncology Group. Am J Clin Oncol 5: 649-655, 1982.

14. Cancer Therapy Evaluation Program 3 (1998) Common Toxicity Criteria, Version 2.0 DCTD, NCI, NIH, DHHS. Revised: 23, 1998

15. Yau T, Yao TJ, Chan P, Wong H, Pang R, Fan ST and Poon RT: The significance of early alpha-fetoprotein level changes in predicting clinical and survival benefits in advanced hepatocellular carcinoma patients receiving sorafenib. Oncologist 16: 1270-1279, 2011.

16. Hutson TE, Bellmunt J, Porta C, Szczylik C, Staehler M, Nadel A, Anderson S, Bukowski R, Eisen T and Escudier B; Sorafenib TARGET Clinical Trial Group: Long-term safety of sorafenib in advanced renal cell carcinoma: Follow-up of patients from phase III TARGET. Eur J Cancer 46: 2432-2440, 2010.

17. Adjei AA, Blumenschein GR Jr, Mandrekar S, Hillman S, Gatzemeier U and Heigener D: Long-term safety and tolerability of sorafenib in patients with advanced non-small-cell lung cancer: A case-based review. Clin Lung Cancer 12: 212-217, 2011.

18. Abdel-Rahman $\mathrm{O}$ and Fouad M: Risk of cardiovascular toxicities in patients with solid tumors treated with sorafenib: An updated systematic review and meta-analysis. Future Oncol 10: 1981-1992, 2014

19. Schmidinger M, Zielinski CC, Vogl UM, Bojic A, Bojic M, Schukro C, Ruhsam M, Hejna M and Schmidinger H: Cardiac toxicity of sunitinib and sorafenib in patients with metastatic renal cell carcinoma. J Clin Oncol 26: 5204-5212, 2008.

20. Duran JM, Makarewich CA, Trappanese D, Gross P, Husain S, Dunn J, Lal H, Sharp TE, Starosta T, Vagnozzi RJ, et al: Sorafenib cardiotoxicity increases mortality after myocardial infarction. Circ Res 114: 1700-1712, 2014 